\title{
Late-onset islet autoimmunity in childhood: the Diabetes Autoimmunity Study in the Young (DAISY)
}

\author{
Brigitte I. Frohnert ${ }^{1}$ - Lisa Ide ${ }^{1}$ • Fran Dong ${ }^{1}$ - Anna E. Barón ${ }^{2}$ • Andrea K. Steck ${ }^{1}$ \\ Jill M. Norris ${ }^{3} \cdot$ Marian J. Rewers ${ }^{1}$
}

Received: 23 November 2016/Accepted: 8 February 2017 /Published online: 17 March 2017

(C) Springer-Verlag Berlin Heidelberg 2017

\begin{abstract}
Aims/hypothesis We sought to assess the frequency, determinants and prognosis for future diabetes in individuals with islet autoimmunity and whether these factors differ depending on the age of onset of islet autoimmunity.

Methods A prospective cohort $(n=2547)$ of children from the general population who had a high-risk HLA genotype and children who had a first-degree relative with type 1 diabetes were followed for up to 21 years. Those with the persistent presence of one or more islet autoantibodies were categorised as early-onset ( $<8$ years of age, $n=143$, median 3.3 years) or late-onset ( $\geq 8$ years of age, $n=64$, median 11.1 years), and were followed for a median of 7.4 and 4.7 years, respectively. Progression to diabetes was evaluated by Kaplan-Meier analysis with logrank test. Factors associated with progression to diabetes were analysed using the parametric accelerated failure time model. Results Children with late-onset islet autoimmunity were more likely to be Hispanic or African-American than nonHispanic white $(p=0.004)$, and less likely to be siblings of individuals with type 1 diabetes $(p=0.04)$. The frequencies of
\end{abstract}

Electronic supplementary material The online version of this article (doi:10.1007/s00125-017-4256-9) contains peer-reviewed but unedited supplementary material, which is available to authorised users.

Brigitte I. Frohnert

brigitte.frohnert@ucdenver.edu

1 Barbara Davis Center for Childhood Diabetes, School of Medicine, University of Colorado, 1775 Aurora Court, A140,

Aurora, CO 80045, USA

2 Department of Biostatistics and Informatics, Colorado School of Public Health, University of Colorado, Aurora, CO, USA

3 Department of Epidemiology, Colorado School of Public Health, University of Colorado, Aurora, CO, USA the HLA-DR3/4 genotype and non-HLA gene variants associated with type 1 diabetes did not differ between the two groups. However, age and HLA-DR3/4 were important predictors of rate of progression to both the presence of additional autoantibodies and type 1 diabetes. Late-onset islet autoimmunity was more likely to present with a single islet autoantibody $(p=0.01)$ and revert to an antibody-negative state $(p=0.01)$. Progression to diabetes was significantly slower in children with late-onset islet autoimmunity $(p<0.001)$.

Conclusions/interpretation A late onset of islet autoimmunity is more common in African-American and Hispanic individuals. About half of those with late-onset islet autoimmunity progress to show multiple islet autoantibodies and develop diabetes in adolescence or early adulthood. Further investigation of environmental determinants of late-onset autoimmunity may lead to an understanding of and ability to prevent adolescent and adult-onset type 1 diabetes.

Keywords Age of diabetes onset - Autoantibodies - Child . Ethnic differences · Islet autoantibody $\cdot$ Racial differences . Type 1 diabetes

\author{
Abbreviations \\ DAISY Diabetes Autoimmunity Study in the Young \\ GADA GAD65 autoantibody \\ IA-2A Islet antigen 2 autoantibody \\ IAA Insulin autoantibody \\ ZnT8A Zinc transporter 8 autoantibody
}

\section{Introduction}

Type 1 diabetes is an autoimmune disease resulting from destruction of the insulin-producing beta cells of the pancreas. 
The development of clinically apparent diabetes is typically preceded by islet autoimmunity [1-3], signalled by the appearance of islet autoantibodies against insulin (IAA) [4], GAD65 (GADA) [5], islet antigen 2 (IA-2A) [6] and zinc transporter 8 (ZnT8A) [7]. Seroconversion may begin in genetically susceptible individuals at an early age. One of the paradigms of the natural history of type 1 diabetes has been that islet autoimmunity rarely develops beyond early childhood. In some studies, a peak incidence of seroconversion has been observed within the first 2 years of life [8-10]; however, seroconversion has been observed well into adolescence and beyond $[11,12]$. Autoantibodies often develop in sequence, rather than at the same time, usually with IAA or GADA appearing first [11] and IA-2A frequently appearing in children who seroconvert early in life [13]. Children who have developed early islet autoimmunity often progress to diabetes at younger ages [14, 15], but it is unclear whether their progression is more rapid or whether it has simply started earlier. Although heterogeneity in islet autoantibody pattern in early- vs late-onset islet autoimmunity has been described, the determinants of these differences are poorly understood.

The Diabetes Autoimmunity Study in the Young (DAISY) has for over 20 years prospectively followed a large cohort of children at increased genetic risk of the development of islet autoimmunity and type 1 diabetes. This cohort study includes a multiethnic and multiracial population of both first-degree relatives of individuals with type 1 diabetes and children recruited from the general population. Our aim was to examine the characteristics of individuals developing autoimmunity at age 8 years or later, compared with children with early-onset autoimmunity in this diverse cohort. We hypothesised that children in our cohort who developed autoimmunity after age 8 years would progress more slowly to type 1 diabetes. Furthermore, we set out to determine which factors were associated with a later onset of autoimmunity to determine the aetiological factors involved in the initiation of islet autoimmunity that leads to type 1 diabetes in young adults. A better understanding of late-onset islet autoimmunity may play a key role in targeting interventions to prevent the development of islet autoimmunity, as well as to slow or halt progression to type 1 diabetes after seroconversion.

\section{Methods}

Study participants DAISY is a prospective cohort study that has followed 2547 children at increased risk of type 1 diabetes for over 20 years (median follow-up 11 years) [16, 17]. Recruitment began in 1993 and includes two groups of children: first-degree relatives of individuals with type 1 diabetes, enrolled between birth and 7 years of age; and participants from the general population, born in Denver, CO, USA, who were identified as having an increased risk of type 1 diabetes based on HLA genotype screening at birth. Of 31,881 newborns screened, all children with HLA genotypes $D R 3 / 4$, $D R 3 / 3$ and $D R 4 / 4$ and a sample of those with $D R 4 / X$ or $D R 3 / X$, in which $X$ was neither $D R 3$ nor $D R 4$, were invited to participate. Newborns with first-degree relatives who had type 1 diabetes were considered to be in the first-degree relative group for the analysis. Of the participants, $3.7 \%$ developed diabetes and met the study endpoint; $85 \%$ of islet antibody-positive individuals without diabetes and $52 \%$ of the original cohort still attend regular study visits. The sample for this study was limited to individuals enrolled in DAISY who developed islet autoimmunity as of 29 September 2015. HLA data were available for all but one of the participants. Data were complete for sex, family history of type 1 diabetes and race/ethnicity. Late-onset islet autoimmunity ( $\geq 8$ years of age was defined based on previous studies [8, 18]. Children who developed autoantibodies before age 8 were defined as 'earlyonset'. The median onset of seroconversion was at 3.3 years of age for children in the early-onset group and 11.1 years for the late-onset group (Table 1). Informed consent was obtained from the parents of all participants. The Colorado Multiple Institutional Review Board approved all study protocols.

Outcome measures Prospective testing for islet autoantibodies was performed at 9 months, 15 months, 24 months and annually thereafter. DAISY participants were tested by radioimmunoassay for GADA, IAA and IA-2A. IAA was measured using a micro-IAA assay as previously described [19]. The initial assay for GADA and IA-2A was a combined radioassay performed in duplicate as previously described [11]. Since January 2010, GADA and IA-2A have been measured using the National Institute of Diabetes and Digestive and Kidney Diseases harmonised assay [20]. All available samples from children who were ever positive for any of the above autoantibodies or who developed type 1 diabetes were tested for ZnT8A as previously described [7]. Additionally, the last sample collected for all 2547 participants was tested for ZnT8A, and if positive, all previous samples were tested to determine the age of seroconversion. Three children who were positive only for ZnT8A were identified in this way. Children who tested positive for any autoantibody were tested more frequently, every 3-6 months. Positive autoantibody tests and $5 \%$ of negative tests were confirmed by blinded quality control. Islet autoimmunity was defined as positivity for one or more islet autoantibodies on two or more consecutive visits or being autoantibody positive with a diagnosis of diabetes at the next visit. Children were diagnosed with diabetes using ADA criteria [21]. The details of the protocols for intensive monitoring and diagnosis of diabetes have previously been described [22]. The dataset reflects follow-up as of 29 September 2015.

Genotyping Polymorphisms of genes implicated in islet autoimmunity and type 1 diabetes risk [23-25] included INS 
Table 1 Characteristics of participants with early- vs late-onset islet autoimmunity

\begin{tabular}{|c|c|c|c|}
\hline Characteristic & $\begin{array}{l}\text { Early-onset }(<8 \text { years }) \\
(n=143)\end{array}$ & $\begin{array}{l}\text { Late-onset ( } \geq 8 \text { years }) \\
(n=64)\end{array}$ & $p$ value \\
\hline Male $(\%)$ & 52 & 47 & 0.52 \\
\hline Non-Hispanic white $(\%)$ & 86 & 69 & 0.004 \\
\hline First-degree relative proband $(\%)$ & 64 & 56 & 0.27 \\
\hline Sibling proband $(\%)$ & 27 & 14 & 0.04 \\
\hline Median age of seroconversion (years) & $3.3(2.0,6.0)$ & $11.1(9.9,13.3)$ & $<0.0001$ \\
\hline$H L A-D R 3 / 4(\%)$ & 34 & 38 & 0.58 \\
\hline Genetic high risk $(\%)$ & 14 & 7 & 0.25 \\
\hline \multicolumn{4}{|l|}{ First antibody } \\
\hline GADA $(\%)$ & 52 & 63 & 0.18 \\
\hline IA-2A $(\%)^{\mathrm{a}}$ & 20 & 16 & 0.42 \\
\hline $\operatorname{IAA}(\%)^{\mathrm{a}}$ & 49 & 28 & 0.005 \\
\hline $\mathrm{ZnT} 8 \mathrm{~A}(\%)^{\mathrm{b}}$ & 31 & 19 & 0.13 \\
\hline $\begin{array}{l}\text { More than one antibody at first positive } \\
\text { sample }(\%)\end{array}$ & 32 & 16 & 0.01 \\
\hline Spreading to other antibodies (\%) & 59 & 42 & 0.02 \\
\hline $\begin{array}{l}\text { Reversion to negative autoantibody status } \\
(\%)\end{array}$ & 35 & 53 & 0.01 \\
\hline Progression to type 1 diabetes (\%) & 49 & 14 & $<0.0001$ \\
\hline $\begin{array}{l}\text { Median follow-up after seroconversion } \\
\text { (years) }^{c}\end{array}$ & $7.4(4.2,10.3)$ & $4.7(2.7,8.7)$ & 0.0027 \\
\hline
\end{tabular}

Genetic high risk was defined by (1) the presence of PTPN22 TT alone or (2) UBASH3A AA in conjunction with $H L A-D R 3 / 4$

$\chi^{2}$ analysis for all frequency results and Wilcoxon rank sum test was used to compare the distribution of median follow-up time between the early and late groups

${ }^{a}$ Missing antibody information for one individual at the seroconversion visit

${ }^{\mathrm{b}}$ Missing antibody information for 47 individuals at the seroconversion visit

${ }^{\mathrm{c}}$ Time from seroconversion to type 1 diabetes or to last visit with interquartile range (rs689), CTLA4 (rs231775), PTPN22 (rs2476601), BACH2 (rs11755527), IL2RA (rs12251307), GLIS3 (rs7020673), GSDM/ORMDL3 (also known as GSDMB; rs2290400), UBASH3A (rs11203203), ERBB3 (rs2292239), CLEC16A (rs12708716), IL27 (rs4788084) and IFIH1 (rs1990760) and were genotyped as previously described [23].

Statistical analysis The incidence of islet autoantibody seroconversion in the DAISY cohort was calculated from the number of children who newly seroconverted to islet autoantibody positivity at each visit using intervals of $0-12$ months, 12-20 months, 20-30 months and yearly intervals thereafter. The number of children followed at each time point was used to calculate the incidence as cases per 1000 person-years, as previously described [8].

SAS version 9.4 (SAS institute, Cary, NC, USA) was used for statistical analyses. The Wilcoxon rank sum test was used to compare distributions of follow-up time between the early- and late-onset groups. Differences in time to the presence of additional autoantibody (i.e. spreading), reversion to antibodynegative status and progression to diabetes were compared using Kaplan-Meier survival analysis with logrank tests.
To examine effects of covariates such as racial/ethnic group and age of seroconversion on time to antibody spread and progression to type 1 diabetes, these variables, in addition to sex, high-risk HLA group and first-degree relative status, were included in a parametric accelerated failure time model [26]. Survival analyses (SAS PROC LIFEREG) were carried out using a parametric model with different underlying time-toevent distributions accounting for right, left and interval censoring. Weibull, exponential, lognormal, logistic and $\gamma$ distributions were considered and fitted to the data, based on $95 \%$ CIs about the respective quantile-quantile plots for the data. The model based on the $\gamma$ distribution showed best fit for spread and progression to diabetes, while the logistic distribution showed the best fit for reversion to antibody-negative status, and the Weibull distribution showed the best fit for progression from multiple autoantibodies to diabetes. Calculation of follow-up time began at seroconversion. The results are presented as acceleration factor estimates with $95 \%$ CIs. These factors summarise the relative time to event compared with a reference group. The acceleration factor $\gamma$ allows an evaluation of the effect of predictor variables on the time to outcome. For example, in individuals compared for the development of diabetes with 
age of seroconversion as a covariate, $\gamma<1$ indicates that older age is harmful to survival free of type 1 diabetes, whereas $\gamma>1$ indicates a benefit on disease-free survival. All tests of hypotheses were performed at a 0.05 level of significance.

\section{Results}

Incidence of islet autoantibodies As of 29 September 2015, persistent islet autoimmunity has developed in 207 DAISY participants, 128 of whom have a first-degree relative with type 1 diabetes. As of September 2015, 79 of these individuals had progressed to clinical diabetes. The incidence of islet autoimmunity appeared to fluctuate from infancy into later childhood, with a broad peak between the ages of 2 and 6 years in first-degree relative children and a later peak at around age 11 years (Fig. 1). The general population children, in contrast, had a more variable incidence. This differs from the more pronounced bimodal pattern noted in previous studies of first-degree relative children [3], who have been shown to have a peak occurring in early childhood followed by a second peak beginning before adolescence. Of note, individual islet autoantibodies had varying profiles at seroconversion, both by age and by first-degree relative/general population status, with IAA showing an early differential peak in first-degree relative children relative to general population children (see electronic supplementary material [ESM] Fig. 1).

\section{Characteristics of individuals with early- vs late-onset islet} autoimmunity Children with late-onset islet autoimmunity were significantly less likely than those with early-onset islet autoimmunity to identify as non-Hispanic white (69\% vs $86 \%, p=0.004)$ (Table 1 ). There were significantly more first-degree relative children among the non-Hispanic white

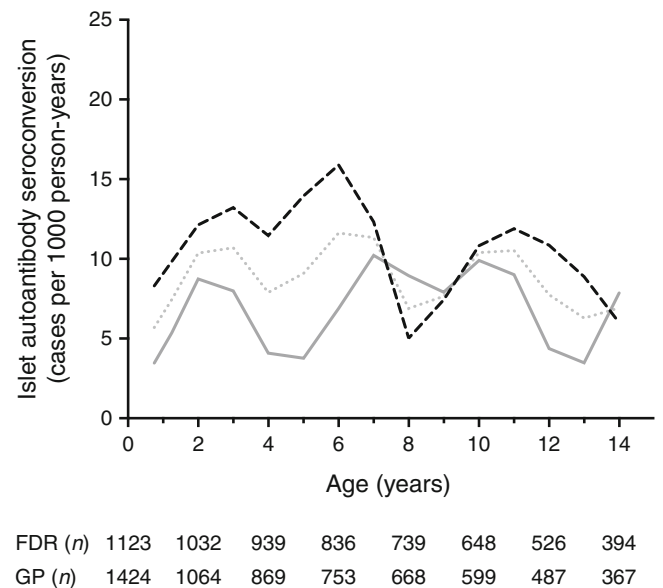

Fig. 1 Age-specific incidence of islet autoantibody seroconversion by family history status. The total numbers of individuals followed at each time point are noted below the graph. Black dashed line, first-degree relative (FDR); solid grey line, general population screened participant (GP); light grey dotted line, incidence for all participants combined children than those of other racial/ethnic groups (67\% vs $40 \%$, $p=0.002$ ); however, both the early and late groups had similar proportions of first-degree relative children relative to those without a family history (Table 1). Of note, the late-onset group was less likely than the early-onset group to have a sibling with diabetes $(14 \%$ vs $27 \%, p=0.04)$. There was no difference in sex distribution between the early- and late-onset islet autoimmunity groups. The assessment of genetic markers showed no difference in the frequency of high-risk HLA type (HLA-DR3/4) the between late- and early-onset groups (Table 1). Previous studies have shown that polymorphisms in PTPN22 and UBASH3A predict time to seroconversion [23]; however, there was no difference in these or other nonHLA risk alleles of genes implicated in development of islet autoimmunity and type 1 diabetes (ESM Table 1). These included BACH2, CTLA4, ERBB3, GLIS3, GSDM/ORMDL3, IL27, IL2RA, INS, PTPN22, UBASH3A and IFIH1. A previous study showed that individuals categorised as having a high genetic risk, as defined by the presence of PTPN22 TT alone or $U B A S H 3 A$ in conjunction with $H L A-D R 3 / 4$, showed a significant difference in time to islet autoimmunity [27]. Although the percentage of individuals with a high genetic risk was greater in the early-onset autoimmunity group, this difference was not significant (Table 1).

The characteristic autoimmune markers also differed between groups (Table 1), as children with late-onset autoimmunity were less likely to present with IAA at seroconversion ( $28 \%$ vs $49 \%, p=0.005$ ), and less likely to initially present with multiple autoantibodies at first positive sample (16\% vs $32 \%, p=0.01)$. When restricted to only participants presenting with a single autoantibody at the time of seroconversion, the late-onset islet autoimmunity group was more likely $(60 \%$, $n=55)$ than the early-onset group (38\%, $n=97 ; p=0.009)$ to be GADA positive. (See ESM Fig. 2 for a detailed summary of autoantibodies at first positive sample.)

Individuals with late-onset islet autoimmunity were less likely to spread to additional autoantibodies when compared with early-onset individuals ( $42 \%$ vs $59 \%, p=0.02$ ), and Kaplan-Meier analysis confirmed that children with a late onset had slower spread to additional autoantibodies than children with an early onset ( $p=0.03$, Fig. 2a). Similarly, individuals with late-onset autoimmunity were less likely to progress to diabetes ( $14 \%$ vs $49 \%, p<0.0001$; Table 1$)$, and this progression was slower ( $p=0.009$, Fig. $2 b$ ). The proportion of firstdegree relatives among early-onset participants progressing to type 1 diabetes was not significantly different from that among late-onset participants ( $66 \%$ vs $78 \%, p=0.47$ ).

Of the participants who achieved multiple autoantibody status (95 early-onset and 26 late-onset), fewer in the lateonset group progressed to diabetes $(27 \%$ vs $69 \%$, $p<0.0001$ ); however, Kaplan-Meier analysis showed no significant difference in progression to diabetes between groups $(p=0.085$; ESM Fig. 3$)$. Time from multiple autoantibody 
a

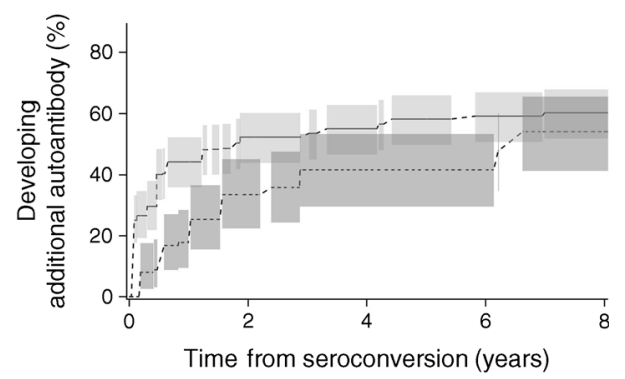

b
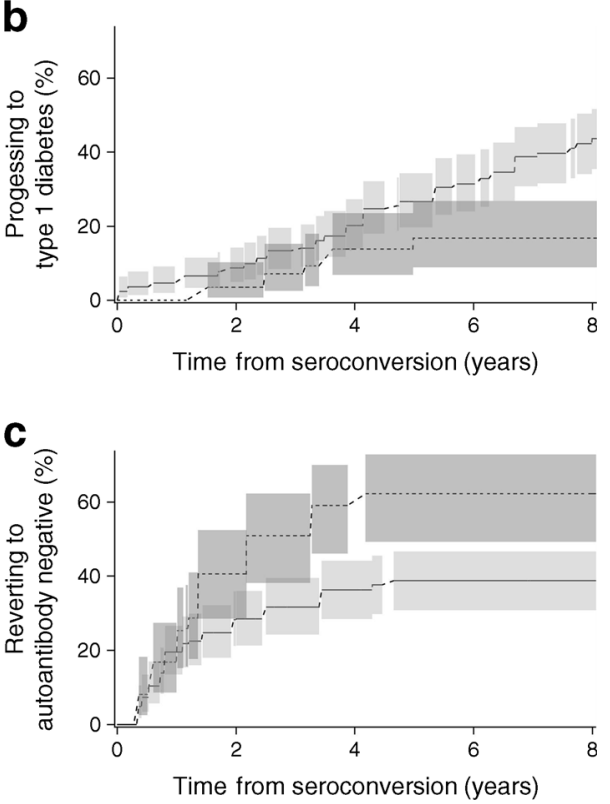

Fig. 2 Kaplan-Meier analysis with logrank test comparison of late-onset $(n=64)$ vs early-onset $(n=143)$ seroconverters. Shaded areas indicate 95\% CIs. Solid line, light grey shading, early-onset; dashed line, dark grey shading, late-onset. (a) Spread to additional autoantibodies in latevs early-onset islet autoimmunity $(p=0.03)$. (b) Time from seroconversion to onset of diabetes $(p=0.009)$. (c) Reversion from islet autoantibody positive to negative status $(p=0.005)$

identification to diabetes did not differ between Hispanic/ African-American $(n=15)$ and non-Hispanic white $(n=105 ; p=0.077$; ESM Fig. 4$)$ individuals.

Although follow-up time following seroconversion was significantly shorter for the late-onset group relative to the early-onset group (4.7 vs 7.4 years $p=0.003$ ), they were nonetheless more likely to revert to negative antibody status $(53 \%$ vs $35 \%, p=0.01)$. Kaplan-Meier analysis of time to reversion showed that individuals in the late-onset group had a significantly greater risk of reversion than early-onset participants ( $p=0.005$; Fig. 2c).

Accelerated failure time analysis In order to examine the effect of various factors on rate of progression from seroconversion to additional autoantibodies or type 1 diabetes, as well as rate of reversion to antibody-negative status, the entire cohort was examined using accelerated failure time analysis with high-risk HLA genotype, age of seroconversion, race/ethnicity, family history of type 1 diabetes and sex as covariates. Following seroconversion, participants with HLA type DR3/4 had a median time to additional autoantibodies that was $74 \%$ shorter than participants with other HLA types (acceleration factor $0.26,95 \%$ CI $0.08,0.84, p=0.02$; Table 2). Increasing age of seroconversion significantly increased time to development of multiple autoantibodies, with a 23\% increase (acceleration factor $1.23,95 \%$ CI 1.10, 1.38, $p<0.001$ ) in median time to development of additional autoantibodies for each additional year of age at seroconversion. Sex, first-degree relative status and race/ethnicity did not influence the time to spread to additional islet autoantibodies.

The $\gamma$ accelerated failure time analysis for progression from seroconversion to diabetes showed that children with HLA type $D R 3 / 4$ had a median time to diabetes that was $64 \%$ shorter than those with other HLA types (acceleration factor $0.36,95 \% \mathrm{CI}$ $0.21,0.61, p<0.001$ ). Older age at seroconversion was also a significant factor for time to progression to diabetes, with a $16 \%$ increase (acceleration factor 1.16, 95\% CI 1.07, 1.26, $p<0.001$ ) in median time to development of diabetes for each additional

Table 2 Characteristics associated with change in rate of spread to additional autoantibodies, progression to type 1 diabetes and reversion to negative autoantibody status after seroconversion $(n=207)$

\begin{tabular}{ll}
\hline Variable & $\begin{array}{l}\text { Acceleration factor } \\
\text { estimate }(95 \% \mathrm{CI})\end{array}$ \\
\hline
\end{tabular}

Spread to additional autoantibodies

$\begin{array}{lll}\text { HLA-DR3/4 (yes vs no) } & 0.26(0.08,0.84) & 0.02 \\ \text { Age at seroconversion (years) } & 1.23(1.10,1.38) & <0.001 \\ \text { Hispanic/AA vs NHW } & 1.81(0.48,6.90) & 0.38 \\ \text { GP vs FDR } & 2.55(0.79,8.25) & 0.12 \\ \text { Sex (female vs male) } & 1.64(0.62,4.29) & 0.32 \\ \text { gression to type 1 diabetes } & & \\ \text { HLA-DR3/4 (yes vs no) } & 0.36(0.21,0.61) & <0.001 \\ \text { Age at seroconversion (years) } & 1.16(1.07,1.26) & <0.001 \\ \text { Hispanic/AA vs NHW } & 2.53(1.10,5.83) & 0.03 \\ \text { GP vs FDR } & 1.21(0.73,1.98) & 0.46 \\ \text { Sex (female vs male) } & 0.98(0.63,1.54) & 0.94\end{array}$

Reversion to autoantibody-negative status

\begin{tabular}{lll}
$H L A-D R 3 / 4$ (yes vs no) & $3.34(1.57,7.11)$ & 0.002 \\
Age at seroconversion (years) & $0.90(0.83,0.97)$ & 0.005 \\
Hispanic/AA vs NHW & $0.31(0.14,0.66)$ & 0.003 \\
GP vs FDR & $0.72(0.36,1.41)$ & 0.33 \\
Sex (female vs male) & $0.73(0.38,1.39)$ & 0.34 \\
\hline
\end{tabular}

Accelerated failure time model analysis for time from seroconversion to spread to additional autoantibodies, progression to diabetes or reversion to autoantibody-negative status

Acceleration factor $>1$ indicates slowing of rate. Acceleration factor $<1$ indicates increased rate of change

AA, African-American; FDR, participant with first-degree relative; GP, general population participant; NHW, non-Hispanic white 
year of age at seroconversion. Hispanic or African-American children also had a median time of progression to diabetes that was 2.5 times longer (acceleration factor $2.53,95 \%$ CI 1.10 , $5.83, p=0.03$ ) than the median for non-Hispanic white children (Table 2). Sex and first-degree relative status did not affect time to progression to diabetes. The accelerated failure time analysis for progression from multiple autoantibody status to diabetes (Table 3) showed that high-risk HLA type continued to shorten time to development of diabetes, with a 50\% decrease in time to progression (acceleration factor $0.50,95 \%$ CI $0.32,0.80$, $p=0.004$ ). Additionally, there was a similar slowing of progression with older age, with an increase in time to diabetes of $17 \%$ with each additional year of age (acceleration factor 1.17, 95\% CI 1.07, 1.28, $p<0.001$ ). No effect of race/ethnicity, sex and first-degree relative status was found.

Finally, accelerated failure time analysis for reversion from seroconversion to autoantibody-negative status showed that $D R 3 / 4$ HLA type was associated with a median time to reversion that was over three times longer than that for other HLA types (acceleration factor 3.34, 95\% CI 1.57, 7.11, $p=0.002$ ). Every additional year of age predicted a $10 \%$ faster time to reversion to antibody-negative status (acceleration factor 0.90 , $95 \%$ CI 0.83, 0.97, $p=0.005$ ). Hispanic or African-American individuals showed a $69 \%$ faster median time to reversion to antibody-negative status (acceleration factor $0.31,95 \% \mathrm{CI}$ $0.14,0.66, p=0.003$ ) than the median for non-Hispanic white individuals (Table 2). Sex and first-degree relative status did not affect time to reversion to autoantibody-negative status.

\section{Discussion}

Our findings demonstrate significant differences in individuals with an early vs late onset of islet autoimmunity and further identify a significant association between later age of seroconversion and slowed development of further autoantibodies and

Table 3 Characteristics associated with progression from multiple autoantibody status to type 1 diabetes $(n=121)$

\begin{tabular}{lll}
\hline Variable & $\begin{array}{l}\text { Acceleration factor } \\
\text { estimate }(95 \% \mathrm{CI})\end{array}$ & $p$ value \\
\hline$H L A-D R 3 / 4$ (yes vs no) & $0.50(0.32,0.80)$ & 0.004 \\
Age at seroconversion (years) & $1.17(1.07,1.28)$ & $<0.001$ \\
Hispanic/AA vs NHW & $1.67(0.74,3.78)$ & 0.22 \\
GP vs FDR & $0.99(0.61,1.61)$ & 0.97 \\
Sex (female vs male) & $0.94(0.60,1.46)$ & 0.77 \\
\hline
\end{tabular}

Accelerated failure time model analysis for time from multiple autoantibody status to progression to diabetes

Acceleration factor $>1$ indicates slowing of progression. Acceleration factor $<1$ indicates increased rate of progression

AA, African-American; FDR, participant with first-degree relative; GP, general population participant; NHW, non-Hispanic white progression to type 1 diabetes. Additionally, later age at seroconversion was associated with an increased rate of reversion to autoantibody-negative status. In participants who became multiple autoantibody positive, the rate of progression to diabetes was slowed with increasing age of initial seroconversion.

We observed the onset of seroconversion beginning to peak between 1 and 2 years of age. However, in addition to the early peak seen in other groups of first-degree relatives [8, 28, 29], we saw a later peak after the age of 8 years in first-degree relative participants, as has previously been described $[3,8,10]$. Participants recruited from the general population had a more evenly distributed incidence over time. In agreement with observations in other populations, we noted that the profile of autoantibodies differed by age of onset, with IAA being more prominent in the early-onset group, and GADA more prominent in the late-onset group. ZnT8A was tested in all serial samples from participants who were positive for another autoantibody and those who later progressed to diabetes. In addition, the last sample collected for all participants was tested for ZnT8A, and if positive, all previous samples were tested. This method has the limitation of potentially missing individuals who seroconverted with only ZnT8A and later reverted to negative autoantibody status, implying that the incidence of ZnT8A and overall islet autoimmunity might be somewhat underestimated.

In addition to a higher risk of progression to diabetes, individuals with early-onset islet autoimmunity more commonly presented with multiple autoantibodies at the first positive sample. This may either be due to the presence multiple autoantibodies at seroconversion or be a consequence of a shorter period of progression from single to multiple autoantibodies, such that the first sample was more likely to miss the short period of single autoantibody positivity. The recent adoption of the term 'stage 1 of type 1 diabetes' to describe multiple islet autoantibody status highlights multiple autoantibody status as a strong predictor of progression to dysglycaemia (stage 2) and symptomatic type 1 diabetes (stage 3) [30].

The incidence of type 1 diabetes is lower in AfricanAmerican and Hispanic individuals compared with nonHispanic white populations. The prevalence of GADA and IA-2A is also lower in African-American children than nonHispanic white children with type 1 diabetes [31]. We have previously shown that non-Hispanic white status plays an agerelated role in risk of seroconversion [32]. This is the first study to show a significant role of racial/ethnic status on reversion to autoantibody-negative status and progression from seroconversion to type 1 diabetes. Of note, race/ethnicity did not appear to be a factor in the progression from multiple autoantibodies to type 1 diabetes. It may be that the decreased rate of progression from seroconversion to diabetes is therefore a result of the increased propensity to revert to autoantibody-negative status. It is important to note, however, that the number of Hispanic and African-American individuals who developed multiple autoantibodies was small, so 
these results must be interpreted with caution. Although the effect of race/ethnicity suggests possible differences in the distribution of genetic risk factors, no differences were noted in markers of genetic risk or in previously described genetic high-risk status [23] between children who developed islet autoimmunity early vs late, although not all known nonHLA SNPs associated with type 1 diabetes were tested in this study. Furthermore the effect of race/ethnicity is potentially confounded by differences in sociodemographic factors, which may affect environmental exposures.

Although there was no difference in first-degree relative status between the two groups, children with early-onset islet autoimmunity were more likely to have siblings with type 1 diabetes. This suggests potential environmental influences for timing of seroconversion, such as early dietary or infectious factors, which would be more likely to be shared by siblings. A variety of early dietary exposures have been implicated in the risk of autoimmunity, including the timing and types of food introduced during infancy [33-35]. Additionally, differences in autoantibody profiles between individuals with earlyand late-onset autoimmunity could indicate that there are different triggers of autoimmunity at different ages or pathogenic differences in acquisition of autoimmunity.

Of note, accelerated failure time modelling showed a significant effect of age at seroconversion on progression to the presence of additional antibodies, reversion to antibodynegative status and time to diabetes (Table 2), an effect that continued to hold true for time from multiple autoantibodies to development of diabetes (Table 3). This observation that a later onset of islet autoimmunity is associated with a slower time to progression is mirrored by a recent study looking at IAA profiles at seroconversion at 9 months and 2 years, showing age- and antibody-related differences in risk of progression [36]. DAISY has previously shown that a family history of type 1 diabetes does not predict the persistence of antibody positivity [37], and this current analysis supports those findings by the lack of a significant effect of first-degree relative status on time to appearance of additional autoantibodies, reversion to antibody-negative status or progression to diabetes.

The limitations of this study include less follow-up after seroconversion for late seroconverters. This may partially explain the lower percentage of individuals with late-onset autoimmunity with spread to additional autoantibodies or progression to diabetes; however, the potential bias of follow-up time was addressed by accounting for censored data in both Kaplan-Meier and accelerated failure time analyses. Both support a role for age of seroconversion in spread to additional autoantibodies and development of diabetes. This cohort requires longer follow-up to fully describe the age of diagnosis of diabetes in late-onset seroconverters. A further limitation is the loss to follow-up of about $44 \%$ of the original cohort, which may add bias to the characteristics of late-onset seroconverters. With regards to genetic analysis, only 11 of the 40-50 non-HLA SNPs associated with susceptibility to type 1 diabetes were analysed in this study owing to incomplete SNP information for all participants, so other non-HLA genes may be involved in later seroconversion. Previous investigation in the DAISY cohort has shown that polymorphisms in PTPN22 and UBASH3A predict time to seroconversion [23]. There was, however, no difference in these genotypes between the early- and late-onset groups, suggesting that analysis restricted to a comparison of earlyvs late-onset islet autoimmunity may be limited in detecting genetic effects. A further weakness is the retrospective nature of the determination of ZnT8A positivity, which could underreport islet autoimmunity if there are individuals who are positive only for ZnT8A and later revert to negative antibody status.

For ongoing study of the pathogenesis of type 1 diabetes and the search for potential interventions, the slower dynamics of late-onset islet autoimmunity will need to be taken into account by screening and prevention programmes. Our findings imply that screening for both islet autoimmunity and progression to diabetes in individuals with islet autoimmunity may need to continue well into or beyond adolescence. Treatment or prevention strategies may need to be targeted based on the age of onset of islet autoimmunity.

In conclusion, our results show important differences in late seroconverters compared with children who develop islet autoimmunity early in childhood. Late-onset in our cohort was associated with more frequent reversion to autoantibody-negative status and slowed progression to type 1 diabetes. While earlyonset islet autoimmunity has been the focus of intense study and may lead to important insight into accelerators of disease, an understanding of late-onset islet autoimmunity may help to unravel the roles of genetics and environment in elucidating triggers for seroconversion as well as rate of progression to type 1 diabetes. Our findings support the hypotheses that either those who experience later onset islet autoimmunity may have been exposed to protective environmental factors that slow or delay both islet autoimmunity and progression to diabetes, or alternatively the constellation of triggers changes with age of seroconversion. Another possibility is the role of the hormonal milieu, including growth hormone and adrenal and gonadal hormones, as a modulating factor for the intensity of responses to triggers. Further investigation into these key factors may lead to the prediction and prevention of type 1 diabetes with an onset into young adulthood and beyond.

Acknowledgements The authors express their gratitude to the children and parents who participated in the study, and thank the DAISY staff for their dedication to this project over the years.

Data availability The datasets generated during and/or analysed during the current study are available from the corresponding author on reasonable request. 
Funding This work was supported by the National Institutes of Health (grants R01 DK32493, DK32083, R01 DK50979, 5K12DK094712), the Diabetes Endocrinology Research Center (P30 DK57516) and the Clinical and Translational Science Award from the National Center for Advancing Translational Sciences to the University of Colorado UL1 TR001082.

Duality of interest statement The authors declare that there is no duality of interest associated with this manuscript.

Contribution statement BIF and LI researched the data, performed statistical analysis and interpretation of the results, and drafted the manuscript. $\mathrm{AB}$ and $\mathrm{FD}$ made major contributions to data analysis and interpretation, and contributed to writing the manuscript. MJR, JN and AKS contributed to conception and design, participated in the data analysis and interpretation, performed critical revision of the article and gave final approval of the article. MJR is the principal investigator of the DAISY study and is the guarantor of this work. All authors approved the final version of the manuscript. As the guarantor of this work, MJR had full access to all the data in the study and takes responsibility for the integrity of the data and the accuracy of the data analysis.

\section{References}

1. Atkinson MA, Eisenbarth GS, Michels AW (2014) Type 1 diabetes. Lancet 383:69-82

2. Bonifacio E (2015) Predicting type 1 diabetes using biomarkers. Diabetes Care 38:989-996

3. Parikka V, Näntö-Salonen K, Saarinen M et al (2012) Early seroconversion and rapidly increasing autoantibody concentrations predict prepubertal manifestation of type 1 diabetes in children at genetic risk. Diabetologia 55:1926-1936

4. Palmer JP, Asplin CM, Clemons P et al (1983) Insulin antibodies in insulin-dependent diabetics before insulin treatment. Science 222: 1337-1339

5. Baekkeskov S, Aanstoot HJ, Christgau S et al (1990) Identification of the $64 \mathrm{~K}$ autoantigen in insulin-dependent diabetes as the GABAsynthesizing enzyme glutamic acid decarboxylase. Nature 347: 151-156

6. Rabin DU, Pleasic SM, Shapiro JA et al (1994) Islet cell antigen 512 is a diabetes-specific islet autoantigen related to protein tyrosine phosphatases. J Immunol 152:3183-3188

7. Wenzlau JM, Juhl K, Yu L et al (2007) The cation efflux transporter $\mathrm{ZnT} 8$ (Slc30A8) is a major autoantigen in human type 1 diabetes. Proc Natl Acad Sci U S A 104:17040-17045

8. Ziegler A-G, Bonifacio E, BABYDIAB-BABYDIET Study Group (2012) Age-related islet autoantibody incidence in offspring of patients with type 1 diabetes. Diabetologia 55:1937-1943

9. Krischer JP, Lynch KF, Schatz DA et al (2015) The 6 year incidence of diabetes-associated autoantibodies in genetically at-risk children: the TEDDY study. Diabetologia 58:980-987

10. Ilonen J, Hammais A, Laine A-P et al (2013) Patterns of beta-cell autoantibody appearance and genetic associations during the first years of life. Diabetes 62:3636-3640

11. Yu L, Rewers M, Gianani R et al (1996) Antiislet autoantibodies usually develop sequentially rather than simultaneously. J Clin Endocrinol Metab 81:4264-4267

12. Colman PG, McNair PD, Gellert S et al (2002) Development of autoantibodies to islet antigens during childhood: implications for preclinical type 1 diabetes screening. Pediatr Diabetes 3:144-148

13. Kimpimäki T, Kulmala P, Savola K et al (2002) Natural history of beta-cell autoimmunity in young children with increased genetic susceptibility to type 1 diabetes recruited from the general population. J Clin Endocrinol Metab 87:4572-4579

14. Hummel M, Bonifacio E, Schmid S et al (2004) Brief communication: early appearance of islet autoantibodies predicts childhood type 1 diabetes in offspring of diabetic parents. Ann Intern Med 140:882-886

15. Steck AK, Johnson K, Barriga KJ et al (2011) Age of islet autoantibody appearance and mean levels of insulin, but not GAD or IA-2 autoantibodies, predict age of diagnosis of type 1 diabetes. Diabetes Care 34:1397-1399

16. Rewers M, Norris JM, Eisenbarth GS et al (1996a) Beta-cell autoantibodies in infants and toddlers without IDDM relatives: Diabetes Autoimmunity Study in the Young (DAISY). J Autoimmun 9:405-410

17. Rewers M, Bugawan TL, Norris JM et al (1996b) Newborn screening for HLA markers associated with IDDM: diabetes autoimmunity study in the young (DAISY). Diabetologia 39:807-812

18. Pflueger M, Seppänen-Laakso T, Suortti T et al (2011) Age- and islet autoimmunity-associated differences in amino acid and lipid metabolites in children at risk for type 1 diabetes. Diabetes 60: 2740-2747

19. Yu L, Robles DT, Abiru N et al (2000) Early expression of antiinsulin autoantibodies of humans and the NOD mouse: evidence for early determination of subsequent diabetes. Proc Natl Acad Sci U S A 97:1701-1706

20. Bonifacio E, Yu L, Williams AK et al (2010) Harmonization of glutamic acid decarboxylase and islet antigen-2 autoantibody assays for National Institute of Diabetes and Digestive and Kidney Diseases consortia. J Clin Endocrinol Metab 95:3360-3367

21. American Diabetes Association (2016) 2. Classification and diagnosis of diabetes. Diabetes Care 39(Suppl 1):S13-S22

22. Stene LC, Barriga K, Hoffman M et al (2006) Normal but increasing hemoglobin A1c levels predict progression from islet autoimmunity to overt type 1 diabetes: Diabetes Autoimmunity Study in the Young (DAISY). Pediatr Diabetes 7:247-253

23. Steck AK, Dong F, Wong R et al (2014) Improving prediction of type 1 diabetes by testing non-HLA genetic variants in addition to HLA markers. Pediatr Diabetes 15:355-362

24. Winkler C, Krumsiek J, Buettner F et al (2014) Feature ranking of type 1 diabetes susceptibility genes improves prediction of type 1 diabetes. Diabetologia 57:2521-2529

25. Törn C, Hadley D, Lee H-S et al (2015) Role of type 1 diabetesassociated SNPs on risk of autoantibody positivity in the TEDDY study. Diabetes 64:1818-1829

26. Hougaard P (1999) Fundamentals of survival data. Biometrics 55: 13-22

27. Romanos J, Rosen A, Kumar V et al (2014) Improving coeliac disease risk prediction by testing non-HLA variants additional to HLA variants. Gut 63:415-422

28. Bonifacio E, Pflüger M, Marienfeld S et al (2008) Maternal type 1 diabetes reduces the risk of islet autoantibodies: relationships with birthweight and maternal HbA1c. Diabetologia 51:1245-1252

29. Achenbach P, Warncke K, Reiter J et al (2004) Stratification of type 1 diabetes risk on the basis of islet autoantibody characteristics. Diabetes 53:384-392

30. Insel RA, Dunne JL, Atkinson MA et al (2015) Staging presymptomatic type 1 diabetes: a scientific statement of JDRF, the Endocrine Society, and the American Diabetes Association. Diabetes Care 38:1964-1974

31. Libman IM, Pietropaolo M, Trucco M et al (1998) Islet cell autoimmunity in white and black children and adolescents with IDDM. Diabetes Care 21:1824-1827

32. Frederiksen BN, Kroehl M, Barón A et al (2015) Assessing agerelated etiologic heterogeneity in the onset of islet autoimmunity. Bio Med Res Int 2015:708289

33. Andrén Aronsson C, Uusitalo U, Vehik K et al (2015) Age at first introduction to complementary foods is associated with 
sociodemographic factors in children with increased genetic risk of developing type 1 diabetes. Matern Child Nutr 11:803-814

34. Norris JM (2010) Infant and childhood diet and type 1 diabetes risk: recent advances and prospects. Curr Diab Rep 10:345-349

35. Virtanen SM, Kenward MG, Erkkola M et al (2006) Age at introduction of new foods and advanced beta cell autoimmunity in young children with HLA-conferred susceptibility to type 1 diabetes. Diabetologia 49:1512-1521
36. Giannopoulou EZ, Winkler C, Chmiel R et al (2015) Islet autoantibody phenotypes and incidence in children at increased risk for type 1 diabetes. Diabetologia 58:2317-2323

37. Barker JM, Barriga KJ, Yu L et al (2004) Prediction of autoantibody positivity and progression to type 1 diabetes: Diabetes Autoimmunity Study in the Young (DAISY). J Clin Endocrinol Metab 89:3896-3902 